\title{
THE MAGNETOSTRICTION AND RESISTANCE OF IRON AND NICKEL.
}

By C. W. Heaps.

HE experiments described in this paper were undertaken with the object of making simultaneous measurements of magnetostriction and resistance in the same specimens of iron and of nickel. The importance of making different experiments on the same specimen is very evident when a comparative study is to be made. The galvanomagnetic properties of a metal are so intimately connected with the other properties such as purity, hardness, crystalline structure, etc., that results of different investigators using different samples cannot be compared with each other with any degree of exactness.

The magnetostriction of iron and nickel has been rather extensively studied by different experimenters, and in a general way all results indicate that a bar of iron has its length increased parallel to the direction of a weak magnetic field and decreased when the field is strong. A bar of nickel suffers a decrease in length for all fields. Different samples of these metals, however, behave differently. Bidwell ${ }^{1}$ states that he has obtained in one specimen of iron a contraction for all field strengths and S. R. Williams ${ }^{2}$ has observed an initial lengthening in the case of nickel. Whether these diversified results are due to differences in the character of the specimen itself or to differences in the uniformity of the magnetic field applied, one can easily see the necessity of making comparative studies from the same sample under the same conditions.

The importance of considering magnetostriction in connection with resistance is made evident by the failure of theory to explain the effect of magnetization upon resistance without bringing into consideration some term depending on the configuration of the molecules in the metal. A magnetic field may cause the free electrons constituting the current to change their paths and hence the resistance is altered. Also, the magnetic field produces a change in the molecular configuration which further affects the resistance. Magnetostriction must also be associated with a change in molecular arrangement, so that by studying magneto-

1 Proc. Roy. Soc., Vol. 56, I894.

2 Phys. Rev., Vol. I, p. 257 , I9I3. 
striction one may hope to obtain a clearer insight into the processes of electric conduction.

The apparatus used is shown in Fig. I. The iron or nickel wires, $A$ and $A^{\prime}$, each about 8 centimeters long and .022 centimeter in diameter were contained in a glass tube, $C C^{\prime}$. By using two wires and a lever, $G$, the effective length of wire was made $16 \mathrm{~cm}$. They were connected as shown so that by joining the copper rods, $K$ and $K^{\prime}$, to a suitable Whaetstone bridge the total resistance of the wire could be accurately measured. Changes of resistance produced by the magnetic field were measured by a method of balancing shunts. Since the resistance of the wires was rather small-less than one ohm-it was necessary to correct carefully for the resistance of the leads. The change of resistance of these copper leads in the magnetic field was neglected. The supporting plugs, $B$ and $B^{\prime}$, were of hard rubber and were firmly cemented into the glass tube. A silk thread extended from the end of $A^{\prime}$ to the lever, $D E F$. One leg of the mirror, $M$, rested on the lever and the other two on a support rigidly attached to the glass tube. The bearings at $G$ and $D$ were of glass on glass. Each bearing was made by melting down the end of a capillary tube till the hole was nearly closed. Into this depression the fine rounded end of the glass axle was fitted. The image of an electric lamp filament was reflected from $M$ upon a scale four or five meters away. Other dimensions were as follows: $F O=0.2 \mathrm{~cm}$., $D E=0.565 \mathrm{~cm} ., E F=3.0$ $\mathrm{cm}$. The magnifying power was calculated to be 32,890 . In order to be sure that no disturbing effects were present the wires, $A$ and $A^{\prime}$, were replaced by silk threads and the apparatus placed in

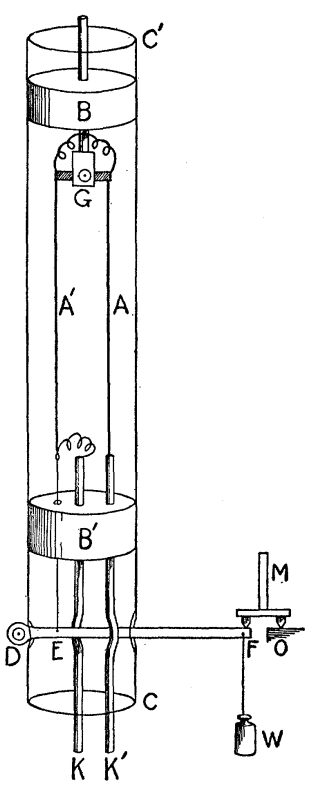

Fig. 1. the magnetic field. No deflection resulted when the magnetic field was thrown on or off. Throughout the experiment the weight $W$ was 40 grams and the temperature was that of the room-about $22^{\circ} \mathrm{C}$.

The longitudinal magnetic field was furnished by a solenoid $15 \mathrm{~cm}$. long and giving a field of roo C.G.S. units for a current of one ampere. No attempt was made to correct for the non-uniformity of the field, though this non-uniformity might be quite a factor in the case of such a short solenoid. However, as the resistance and the magnetostriction could be measured without moving the apparatus, any irregularity in the field would probably not affect the value of the data as far as purposes of comparison are concerned. 
For producing the transverse field a Weiss electromagnet with polepieces $10 \mathrm{~cm}$. in diameter was used. The glass tube of the apparatus was made to have a small diameter so that the pole-pieces could be brought as close together as $1.7 \mathrm{~cm}$. Of course inaccuracy in adjusting the apparatus would introduce a small longitudinal component of magnetization. Jones and Malam ${ }^{1}$ have shown that an error of one degree in adjusting the apparatus may affect the results obtained to a slight extent. However, such small errors in the present experiment would not affect comparative study. The magnetic field was measured by the use of a bismuth spiral. The chemical composition of the metals was not definitely determined. Soft Norway iron wire was used in one case. The nickel was of the ordinary kind furnished by Eimer and Amend.

In taking the data it was found inadvisable to make simultaneous observations of resistance change and length change, because the current

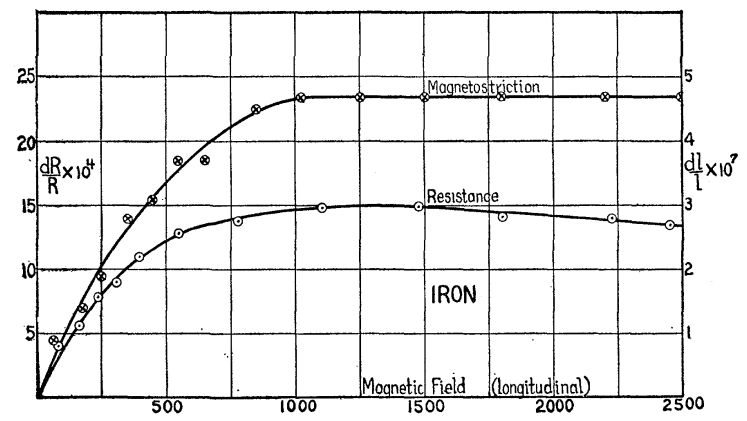

Fig. 2.

through the wire produced disturbing heat effects. Consequently a series of readings for magnetostriction was taken and immediately afterwards a series for resistance. Hysteresis effects were not studied, the observations beginning in every case with a maximum field and ending with the weak field. In the case of nickel the specimen was moved and readjusted between the series of observations, but the accuracy of readjustment was such that any error introduced would be very slight. When iron was used care was taken not to touch the apparatus between observations.

The curves shown are plotted from the data obtained, $d l / l$ being change of length divided by length, and $d R / R$ change of resistance divided by resistance. The external magnetic field measured in gauss is plotted without correction being made for the demagnetizing effect of the metal wires. If the resistance increased, values of $d R / R$ are plotted above

${ }^{1}$ Phil. Mag., Vol. 27, I9I4, p. 649. 
the axis of abscissæ; while if the length decreased $d l / l$ is plotted above the axis on the same diagram except in Fig. 5, where $d l / l$ reprseents a contraction. The resistance curves are similar in every respect to the ones previously obtained by the writer. ${ }^{1}$

The drop in the resistance curve of Fig. 2 is due to transverse magnetization. It has been shown ${ }^{2}$ that with the pure metal and uniform fields there is no initial increase of resistance in a transverse field for either iron or nickel. It is as yet difficult to say whether impurities or a longitudinal component of magnetization is responsible for the initial increase observed in iron in the present experiments.

The magnetostriction curves differ in some respects from the majority

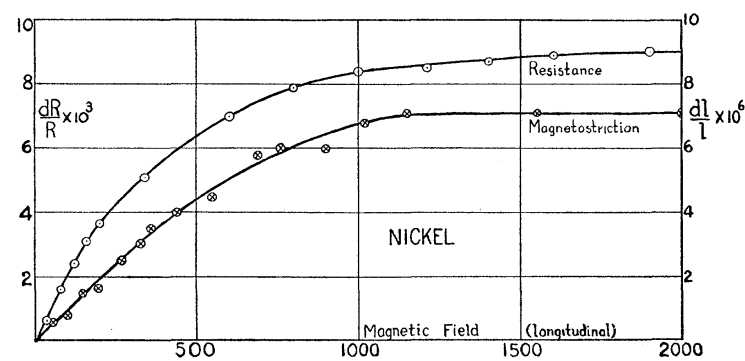

Fig. 3.

of the published results. Iron was found to contract for all values of the longitudinal magnetic field. This contraction is in agreement with one of Bidwell's curves but is at variance with the results of other workers. The transverse field also produced a contraction, though it is possible that in larger fields an elongation might result, as the course of the curve indicates that "saturation" has not been reached at 12,000 . In nickel the transverse field produced an elongation and the longitudinal field a contraction.

In Figs. 2 and 3 there is remarkable parallelism between the resistance curves and the magnetostriction curves. An increase of resistance is associated with a decrease of length, and it seems probable that whatever causes operate to change the resistance also operate according to much the same law to change the length of the conductor. According to Drude's theory of metallic conduction the electric current is given by

$$
I=\frac{\mathrm{I}}{2} N \frac{e^{2}}{m} X \frac{L}{u},
$$

where $N$ is the number of electrons per unit volume, $X$ is the electric force, $u$ the average velocity of the electrons, and $L$ the mean free path.

1 Phil. Mag., Dec., r9II, p. 900.

2 Jones and Malam, 1. c. R. A. Heising, Phys. Rev., Vol. IV., 4, I9I4, p. 3I5. 
This equation is usually assumed to hold true even in the newer theories of metallic conduction, where application of the quantum hypothesis is made. Presumably, by changing the arrangement of the molecules, the longitudinal magnetic field affects $L$. If $L$ is made smaller the resistance is increased. Let $I^{\prime}$ be the current when the magnetic field acts. Then

$$
I^{\prime}=\frac{\mathbf{I}}{2} N \frac{e^{2}}{m} X \frac{L^{\prime}}{u}=\frac{X}{R^{\prime}},
$$

where $L^{\prime}$ is the new free path and $R^{\prime}$ the new resistance. Subtracting this equation from the first gives

or

$$
\frac{\mathrm{I}}{R}-\frac{\mathrm{I}}{R^{\prime}}=\frac{\mathrm{I}}{2} \frac{N e^{2}}{m u}\left(L-L^{\prime}\right)
$$

$$
\frac{d R}{R}=\frac{d L}{L} .
$$

Here $d L$ is the decrease in $L$ while $d R$ is the increase in $R$. It seems reasonable to suppose that when the intermolecular distances are made smaller the length of the conductor as a whole should decrease proportionally. That is, we might expect

$$
\frac{d L}{L}=C \frac{d l}{l},
$$

where $C$ is a constant and $l$ is the length of the conductor. Hence,

$$
\frac{d R}{R}=C \frac{d l}{l} .
$$

In Fig. 6 the values of $d R / R$ are plotted against corresponding values of $d l / l$. In the case of iron a straight line results as is to be expected from the above theoretical considerations. No values are taken from the resistance curve for fields above $\mathrm{I}, 200$, as transverse magnetization causes a drop above that point. The curve of Fig. 6 for nickel is also a straight line for large fields but for small fields the law of direct proportionality no longer holds. W. A. Jenkins ${ }^{1}$ has studied magnetostriction and resistance in different specimens of nickel and he comes to the conclusion that $d R / R$ is proportional to $\sqrt{ } \overline{d l / l}$ for fields below roo C.G.S. units. For larger fields than this the law will not hold. Data taken from the observations of other experimenters do not support Jenkins' conclusion, though, as was pointed out above, data obtained from different samples may give different results. The crystalline structure of a nickel rod may be quite different from that of a drawn wire. However

\footnotetext{
1 Phil. Mag., Vol. 27, I9I4, p. 73 r.
} 
it seems possible that in the case of nickel one cannot assume the change of intermolecular distance for small magnetic fields to be proportional to the total change of length as in iron.

In the case of a transverse magnetic field two factors enter to change the resistance-(I) the molecular rearrangement, and (2) the direct

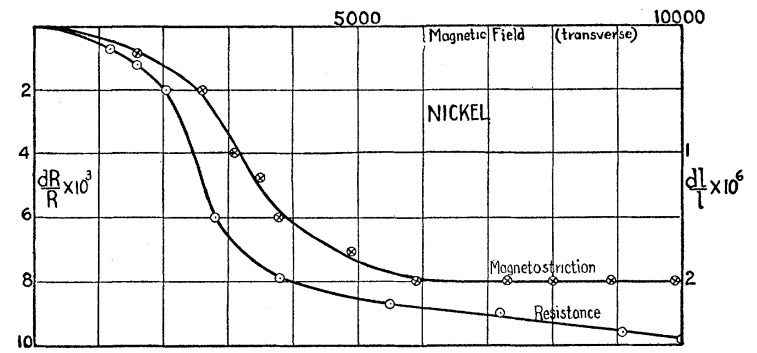

Fig. 4.

deflection of the electrons by the magnetic field. ${ }^{1}$ We would therefore expect the magnetostriction and resistance curves of Figs. 4 and 5 to resemble each other only when term (I) is large compared with term (2). This seems to be the case in the nickel specimen studied, though even here the effect of term (2) is evident. When the magnetic field is made

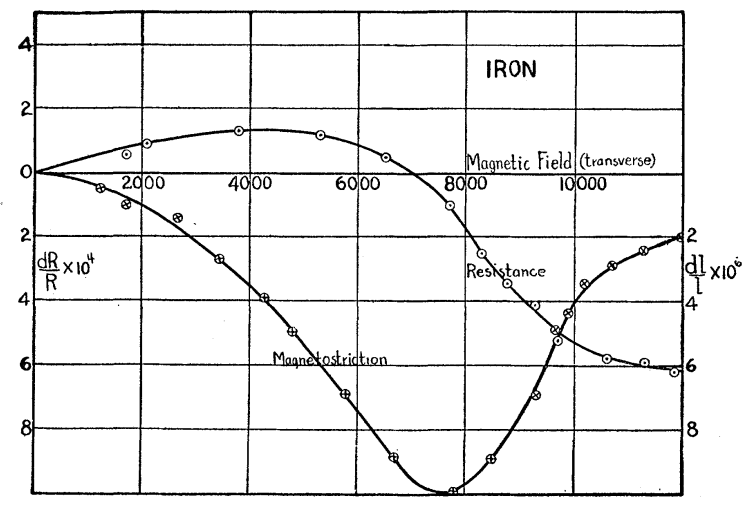

Fig. 5.

as large as 6,000 the molecular rearrangement is complete and the length ceases to increase. The resistance, however, continues to decrease slightly as the field increases, and this decrease must be attributed to the magnetic deflection of the electrons.

1 For a longitudinal field (2) also produces "an effect, but it is smaller than for a transverse field. The writer has calculated the magnitude of (2) to be $\frac{\mathrm{I}}{36} H^{2} \frac{e^{2}}{m} T^{2}$ for a longitudinal field. For a transverse field it is $\frac{\mathrm{r}}{4} H^{2} \frac{e^{2 \mid}}{m} T^{2}$. (E. P. Adams, Phys. Rev., May, I907, p. 428 .) 
The curves for iron (Fig. 5) show that the molecular rearrangement is not complete for fields as large as 12,000 . That is, the length change is still dependent on the magnetic field when it has that value. This would seem rather surprising were it not probable that the intensity of magnetization and not the magnetic field is the factor which determines the form of the curves, and it is much more difficult to magnetize wires transversely than longitudinally. The complex nature of the magnetostriction curve for iron would lead one to expect a complex resistance curve, such as is shown, especially if the two factors mentioned above as determining the resistance change are simultaneously operative. It is perhaps significant that as long as a transverse magnetic field produces an increase in the resistance of iron the contraction of the iron increases. When the resistance change becomes negative the contraction begins to decrease in magnitude.

In all of the curves except those of Fig. 5 the resistance increase is coupled with a length decrease, and vice versa, and for iron the tendency is towards this relation as is pointed out above. Guthe and Austin ${ }^{1}$ have found an increase in the length of Heusler alloy placed in a magnetic field. The previous experiments ${ }^{2}$ of the writer on this metal have shown that the resistance decreases, and the curve obtained is similar to the

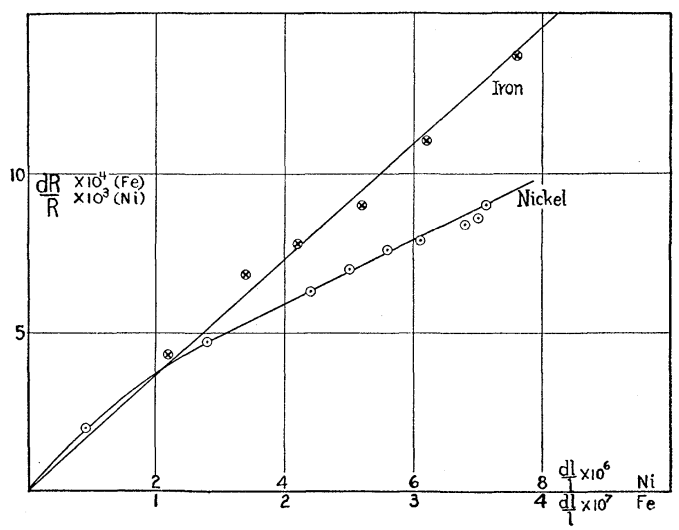

Fig. 6.

magnetostriction curves of Guthe and Austin. Though a law of direct proportionality may not hold good in all cases it is noteworthy that those metals showing large magnetostriction also show a large resistance change. The magnitude of the two effects follows the order: nickel, Heusler alloy, iron. If the analogy holds good for other metals we might expect $d l / l$ in the non-magnetic metals to be of the order $\mathrm{IO}^{-8}$ or $\mathrm{IO}^{-9}$.

${ }^{1}$ Bull. Bur. Standards, 2, 297, 1906.

2 'Phil. Mag. Dec., I9II, p. 900. 
The substitution of experimental data in any of the formulæ derived from our present theories of electric conduction cannot carry one very far on the road towards proving these theories. The mathematical expression for the resistance change in a transverse field is

$$
\frac{d R}{R}=\frac{d T}{T}-\frac{\mathrm{I}}{4} H^{2} \frac{e^{2}}{m} T^{2}, 1
$$

where $T$ is the free period of the electrons in the metal. Various attempts have been made to explain experimental results using this equationJenkins finding it unsatisfactory and Jones and Malam meeting with fair success. It is doubtful whether sufficient data can be obtained to justify numerical substitutions in the above equation since $H$ cannot properly be taken as the external magnetic field or the magnetic induction. It is more proper to consider it as corresponding to the "molecular field" of Weiss's magnetic theory, and the changes produced in this molecular field by an external field must be of a very complex character.

The data obtained in this experiment permit of a comparison being made between the longitudinal and transverse magnetostrictive effects, though as has been pointed out by S. R. Williams, the crystalline structure parallel to the length of a wire is different from that perpendicular to the length, so that comparisons cannot be carried too far. If we neglect this factor we find that iron contracts at right angles to the magnetic field as well as parallel to the field. The resulting decrease of volume per unit volume is of the order $\mathrm{IO}^{-6}$. Nickel expands perpendicularly to the field and contracts in the direction of the field. The calculated maximum decrease in volume of nickel is $3 \times \mathrm{IO}^{-6}$. These conclusions are in contradiction to the experiments of Nagaoka and Honda who found an increase in volume for both iron and nickel.

S. R. Williams, in a series of recent articles, has sought to attribute magnetostriction to the orientation of molecular magnets having the form of oblate spheroids. For this theory to hold good transverse elongation of a conductor would have to be associated with longitudinal contraction, and vice versa. In nickel this is the case, but in iron the phenomena seem to be of too complex a nature to allow of such a simple explanation. It is probable that some sort of structural modification takes place in which the molecular grouping is changed by the magnetic field, and this changed molecular grouping may be taken in connection with a theory of oblate spheroids to explain most of the phenomena observed.

1 E. P. Adams, Phys. Rev., May, r907, p. 428. 
Summary.

I. The specimen of iron was found to contract when magnetized either transversely or longitudinally.

2. The specimen of nickel contracts in the direction of a magnetic field and expands perpendicularly to the magnetic field.

3. For a longitudinal field the magnetostriction and resistance curves are found to be very similar-in iron the resistance increase is proportional to the contraction.

4. For a transverse field the magnetostriction and resistance curves of nickel are very similar, showing a close relationship between the two effects. In iron the relationship is also evident, but is more complex than in the case of nickel. A decrease of resistance is associated with a diminution of magnetostriction, and an increase of resistance with an increase of magnetostriction.

5. Except with iron in a transverse field contraction is always coupled with an increase of resistance and expansion with a decrease of resistance.

6. Application of the electron theory to the experimental results is as satisfactory as could be expected, though ignorance of the laws governing intermolecular actions in different metals prevents to a considerable extent the testing out of any theory.

The Rice Institute, Houston, Texas. 